\title{
Contribuição ao Estudo Psicossocial da Adolescência
}

\author{
Clair $\mathrm{T} r$ zinha Lazzar $\mathrm{tti}^{1}$ \\ osir s P r ira d Andrad ${ }^{2}$ \\ Claud $\mathrm{t} \quad$ giani $^{3}$
}

\section{ESUMO}

LA A TT C. T.; AND AD . P.; G AN C. Contribuição ao studo psicossocial da adol scência. R.B.S.H. 1(2): 1990.

No D partam nto d Tocogin cologia da Univ rsidad $\mathrm{F} d$ ral do Paraná foi criado o AMA - At ndim nto Multidisciplinar ao Adol sc nt - com a finalidad $\mathrm{d}$ of $\mathrm{r} \mathrm{c} \mathrm{r}$ um at ndim nto sp cífico a ssa faixa tária. No s tor d Psicologia do AMA foram aplicados 120 qu stionários s ndo 60 para adol sc nt s grávidas 60 para não grávidas $t$ ndo por formalidad $r$ alizar uma avaliação psicossocial das $m$ smas. D ntr os principais dados obtidos d stacamos:

1. ntr as grávidas $80 \%$ tinha idad ntr 1617 anos;

2. $95 \%$ das grávidas $805 \%$ das não grávidas com çaram a namorar ant $\mathrm{s}$ dos 16 anos d idad ;

3. $585 \%$ das grávidas iniciaram atividad $\mathrm{s}$ xual ant $\mathrm{s} d$ compl tar 16 anos das não grávidas 47\%;

4. $84 \%$ das grávidas $675 \%$ das não grávidas tinham ap nas um parc iro;

5. Com r lação à s nsação d praz $r$ durant o ato $s$ xual ap nas $47 \%$ das grávidas $38 \%$ das não grávidas r f riram s nti-lo;

\footnotetext{
1. Psicóloga.

2. Gin cologista.

3. Gin cologista.

Trabalho r alizado na Disciplina d produção Humana- D partam nto d Tocogin cologia da Univ rsidad F d ral do Paraná-UFPr-Curitiba $\mathrm{P}$

c bido m 30.0490

Aprovado m 14.05.90
} 
6. A quase totalidade das adolescentes referiram conhecer os métodos anticoncepcionais, mas entre as grávidas apenas $31,5 \%$ referiram já ter utilizado;

7. $80 \%$ das grávidas viviam junto com o companheiro $(36.5 \%$ casadas e 43,5 amasiadas) e 70\% das não grávidas não viviam com nenhum companheiro;

8. As pacientes grávidas referiram bom relacionamento com o companheiro em $76 \%$ das vezes, com a mãe em $60 \%$ e com o pai em apenas $23 \%$.

\section{SUMMARY}

LAZZARETTI, C. T.; ANDRADE, R. P.; REGIANI, C. Contribution to the psychological and social study of adolescence. RB.S.H. 1(2): 1990.

In the Department of Obstetrics and Gynecology of the Federal University of Paraná, Brazil, it was created the AMA - Multidisciplinary Assistance to Adolescent - to offer a specific assistance to this population. In the psychologic sector, 120 questionaires were applicated to 60 pregnant and to 60 non pregnant adolescents. The results are:

1. $80 \%$ of the pregnant were between 16 and 17 years old;

2. $95 \%$ of the pregnants and $80,5 \%$ of the non pregnants had a boyfriend before the age of 16 ;

3. $58,5 \%$ of the pregnants began sexual activity before the 16 and between the non pregnants, $47 \%$;

4. $84 \%$ of the pregnants and $67,5 \%$ of the non pregnants had only one partner;

5. Only $47 \%$ of the pregnants and $38 \%$ of the non pregnants had pleasure during the intercourse;

6 . The great majority of the adolescents knew the contraceptive methods, but only $31,5 \%$ of the pregnants had already used them:

7. $80 \%$ of the pregnants were living with their partners $(36,5 \%$ married) and $70 \%$ of the non pregnants were living without a partner;

8. $76 \%$ of the pregnants said they had good relationship with their partners, $60 \%$ with their mothers and only $23 \%$ with their fathers.

\section{INTRODUÇAO}

A partir de agosto de 1988, no Departamento de Tocoginecologia da Universidade Federal do Paraná, foi criado o AMA Atendimento Multidisciplinar ao Adolescente - em convênio com a Fundação Pathfinder. 
Essa iniciativa visou um atendimento global a personalizado, em local específico, ao adolescente, realizado por uma equipe especializada, composta de médicos, psicóloga, assistente social, auxiliar de enfermagem e secretária.

Foi estabelecido que no AMA seriam atendidas adolescentes grávidas até no máximo 17 anos de idade e não grávidas até 19 anos, inclusive.

A adolescência é uma fase do desenvolvimento humano entre a infância e a idade adulta quando ocorrem uma série de rápidas transformações físicas, psicológicas e sociais. Em função deste momento, em que se apresentam tantas mudanças em seu desenvolvimento, é de fundamental importância que procuremos conhecer as características da adolescente.

Com essa finalidade, no setor de psicologia do AMA foi desenvolvido um questionário, aplicado às adolescentes grávidas e não grávidas, para avaliar as características psicossociais.

\section{MATERIAL E MÉTODO}

As adolescentes participantes desta avaliação foram atendidas no ambulatório do AMA, na maternidade do Hospital das Clínicas, no período de agosto de 1988 até junho de 1989.

Os questionários foram todos aplicados pela mesma pessoa do setor de Psicologia e, na maioria das vezes, após as adolescentes já terem sido atendidas em outro setor do AMA.

Os questionários aplicados constavam de 90 bens de perguntas abertas. O exame de estado mental foi também realizado no decorrer da entrevista.

Entre as adolescentes nas quais se aplicou o questionário, foi realizada uma divisão em doffs grupos:

- Grupo I - 60 adolescentes grávidas.

- Grupo II - 60 adolescentes não grávidas.

A análise dos dados dos dois grupos estudados foi realizada no Departamento de Tocoginecologia da UFPr, separadamente, e, em determinadas situações, comparativamente.

\section{OBJETIVOS}

Os objetivos desta avaliação foram:

1. Conhecer as características das adolescentes em função das variáveis: faixa etária, idade de início de namoro, idade de 
início da atividade sexual, conhecimento e utilização de métodos anticoncepcionais.

2. Conhecer as características do relacionamento com o companheiro e com os pais.

\section{RESULTADOS}

Entre as adolescentes grávidas entrevistadas (Grupo I), a grande maioria (80\%) situava-se na faixa etária dos $16-17$ anos; $13,5 \%$ tinha 15 anos e 6,5\%, 13 e 14 anos de idade. Entre as não grávidas (Grupo II), 71,5\% tinha idades compreendidas entre os 16 e os 19 anos (Tabela 1).

Tabela 1 - Distribuição das adolescentes pela faixa etária.

\begin{tabular}{c|c|c|c|c}
\hline \multirow{2}{*}{ Idade } & \multicolumn{2}{|c|}{ Grupo I } & \multicolumn{2}{c}{ Grupo II } \\
\cline { 2 - 5 } & No & \% & No & \% \\
\hline 11 & - & - & 1 & 1,5 \\
12 & - & - & 2 & 3,5 \\
13 & 1 & 1,5 & 1 & 1,5 \\
14 & 3 & 5,0 & 6 & 10,0 \\
15 & 8 & 13,5 & 7 & 12,0 \\
16 & 22 & 36,5 & 12 & 20,0 \\
17 & 26 & 43,5 & 11 & 18,5 \\
18 & - & - & 11 & 18,5 \\
19 & - & - & 9 & 14,5 \\
\hline Total & 60 & 100,0 & 60 & 100,0 \\
\hline
\end{tabular}

Com relação à idade de início de namoro (Tabela 2), podemos observar que $15 \%$ do Grupo I e $12 \%$ do Grupo II iniciaram namoro entre 9 e 12 anos de idade; entre 13 e 14 anos de idade, $55 \%$ do Grupo I e $47 \%$ do Grupo II; com 15 ou mais anos de idade, $30 \%$ do Grupo I e 41 \% do Grupo II.

No que se refere ao início da atividade sexual, $15 \%$ do Grupo 1 e $9 \%$ do Grupo II tiveram a primeira experiência entre 11 e 13 anos de idade; $43,5 \%$ do Grupo I e 38\% do Grupo II, entre 14 e 15 anos de idade; $41,5 \%$ do Grupo I e 53\% do Grupo II, entre 16 e 17 anos. 
Tabela 2 - Distribuição das adolescentes segundo a idade de início de namoro e de início de atividade sexual.

\begin{tabular}{c|c|c|c|c|c|c|c|c}
\hline \multirow{2}{*}{ Idade } & \multicolumn{4}{|c|}{ Início Namoro } & \multicolumn{3}{c}{ Início Atividade Sexual } \\
\cline { 2 - 9 } & \multicolumn{2}{|c|}{ Grupo I } & \multicolumn{2}{c|}{ Grupo II } & \multicolumn{2}{c|}{ Grupo I } & \multicolumn{2}{c}{ Grupo II } \\
\cline { 2 - 9 } & No & $\%$ & No & $\%$ & No & $\%$ & No & $\%$ \\
\hline \multirow{2}{*}{09} & 1 & 1,5 & - & - & - & - & - & - \\
10 & 2 & 3,5 & 1 & 2,0 & - & - & - & - \\
11 & 1 & 1,5 & - & - & 1 & 1,5 & - & - \\
12 & 5 & 8,5 & 5 & 10,0 & 1 & 1,5 & - & - \\
13 & 14 & 23,5 & 6 & 12,0 & 7 & 12,0 & 3 & 9,0 \\
14 & 19 & 31,5 & 18 & 35,0 & 10 & 17,0 & 2 & 6,0 \\
15 & 15 & 25,0 & 11 & 21,5 & 16 & 26,5 & 11 & 32,0 \\
16 & 3 & 5,0 & 7 & 13,5 & 22 & 36,5 & 9 & 26,5 \\
17 & - & - & 2 & 4,0 & 3 & 5,0 & 9 & 26,5 \\
18 & - & - & - & - & - & - & - & - \\
19 & - & - & 1 & 2,0 & - & - & - & - \\
\hline \multirow{2}{*}{ Total } & 60 & 100,0 & $51 *$ & 100,0 & 60 & 100,0 & $34 * *$ & 100,0 \\
\hline
\end{tabular}

* 9 pacientes do Grupo II não namoraram.

** 26 pacientes do Grupo II não iniciaram atividade sexual.

É importante ressaltar que os resultados percentuais do Grupo II com relação aos dois últimos dados referem-se apenas às adolescentes que iniciaram namoro (51) e atividade sexual (34).

Na Tabela 3 avalia-se a variação de parceiros de atividade sexual. Observa-se que $84 \%$ do Grupo 1 e $67,5 \%$ do Grupo II (consierando-se as 34 pacientes que já iniciaram atividade sexual) têm ou tiveram um único parceiro; $14,5 \%$ de ambos os grupos, dois parceiros.

Os dados da questão formulada sobre a presença ou a ausência de sensação de prazer no coito são mostrados na Tabela 4. Do Grupo I, $47 \%$ e, do Grupo II, 38\% referiram sentir prazer, enquanto que $53 \%$ do Grupo I e $50 \%$ do Grupo II informaram que não sentiam nenhum prazer na relação sexual.

Os métodos contraceptivos são conhecidos por $91,5 \%$ das adolescentes do Grupo I e 92,5\% do Grupo II. Quanto à utilização, apenas $31,5 \%$ das grávidas já haviam usado algum tipo de método anti- 
Tabela 3 - Distribuição das adolescentes segundo a variação de parceiros.

\begin{tabular}{c|c|c|c|c}
\hline Parceiros & \multicolumn{2}{|c|}{ Grupo I } & \multicolumn{2}{c}{ Grupo II } \\
\hline No & No & \% & No & $\%$ \\
\hline 1 & 50 & 84,0 & 23 & 67,5 \\
2 & 9 & 14,5 & 5 & 14,5 \\
3 & 1 & 1,5 & 3 & 9,0 \\
4 & - & - & 2 & 6,0 \\
5 & - & - & - & 3,0 \\
6 & - & - & 1 & 100,0 \\
\hline Total & 60 & 100,0 & $34^{*}$ & \multicolumn{2}{c}{}
\end{tabular}

* 26 pacientes do Grupo II não iniciaram atividade sexual.

Tabela 4 - Distribuição das adolescentes segundo a sensação de prazer na atividade sexual.

\begin{tabular}{l|c|c|c|c}
\hline \multirow{2}{*}{$\begin{array}{c}\text { Sensação } \\
\text { de Prazer }\end{array}$} & \multicolumn{2}{|c|}{ Grupo I } & \multicolumn{2}{c}{ Grupo II } \\
\cline { 2 - 5 } & No & $\%$ & No & $\%$ \\
\hline Sim & 26 & 47,0 & 13 & 38,0 \\
Não & 29 & 53,0 & 17 & 50,0 \\
Às vezes & - & - & 4 & 12,0 \\
\hline Total & $55^{*}$ & 100,0 & $34^{* *}$ & 100,0 \\
\hline
\end{tabular}

* 5 pacientes do Grupo I não responderam.

** 26 pacientes do Grupo II não iniciaram atividade sexual.

concepcional. Entre as 34 adolescentes não grávidas que já tinham tido atividade sexual, 28 usam ou já usaram algum método anticoncepcional (Tabela 5).

Considerando, como situação conjugal, o casal viver ou não junto, constatamos que, no Grupo I, $36,5 \%$ e, no Grupo II, $16,5 \%$ eram casadas; $43,5 \%$ e $13,5 \%$, respectivamente, amasiadas; $20 \%$ das grávidas e $68,5 \%$ das não grávidas eram solteiras (Tabela 6 ). 
Tabela 5 - Distribuição das adolescentes segundo conhecimento e utilização dos métodos contraceptivos.

\begin{tabular}{|c|c|c|c|c|c|c|c|c|}
\hline \multirow{3}{*}{$\begin{array}{c}\text { Métodos } \\
\text { Anti- } \\
\text { concepcionais }\end{array}$} & \multicolumn{4}{|c|}{ Grupo I } & \multicolumn{4}{|c|}{ Grupo II } \\
\hline & \multicolumn{2}{|c|}{ Conhecimento } & \multicolumn{2}{|c|}{ Utilização } & \multicolumn{2}{|c|}{ Conhecimento } & \multicolumn{2}{|c|}{ Utilização } \\
\hline & №. & $\%$ & No & $\%$ & No & $\%$ & No & $\%$ \\
\hline Sim & 55 & 91,5 & 19 & 31,5 & 51 & 92,5 & 28 & 51,0 \\
\hline Não & 5 & 8,5 & 41 & 68,5 & 4 & 7,5 & 27 & 49,0 \\
\hline Total & 60 & 100,0 & 60 & 100,0 & $55^{*}$ & 100,0 & $55^{*}$ & 100,0 \\
\hline
\end{tabular}

* 5 pacientes do Grupo II não responderam.

** 26 pacientes do Grupo II não iniciaram atividade sexual.

Tabela 6 - Distribuição das adolescentes segundo a situação conjugal.

\begin{tabular}{l|c|c|c|c}
\hline \multirow{2}{*}{$\begin{array}{c}\text { Situação } \\
\text { Conjugal }\end{array}$} & \multicolumn{2}{|c|}{ Grupo I } & \multicolumn{2}{c}{ Grupo II } \\
\cline { 2 - 5 } & No & \% & No & \% \\
\hline Casadas & 22 & 36,5 & 10 & 16,5 \\
Amasiadas & 26 & 43,5 & 8 & 13,5 \\
Solteiras & 12 & 20,0 & 41 & 68,5 \\
Separadas & - & - & 1 & 1,5 \\
\hline Total & 60 & 100,0 & 60 & 100,0 \\
\hline
\end{tabular}

O relacionamento com os pais e com o companheiro é avaliado na Tabela 7. Ressaltamos que $60 \%$ das grávidas tinham bom rela cionamento com a mãe e $76 \%$ delas também tinham bom relacionamento com o companheiro. Com o pai, $63,5 \%$ das grávidas referiram relacionamento conflituoso. Este questionamento dizia respeito ao relacionamento com os pais antes da instalação da gravidez. Entre as não grávidas, cerca da metade relatou bom relacionamento com a mãe, com o pai e com o companheiro (neste último considera-se as 21 adolescentes que tinham companheiro). 


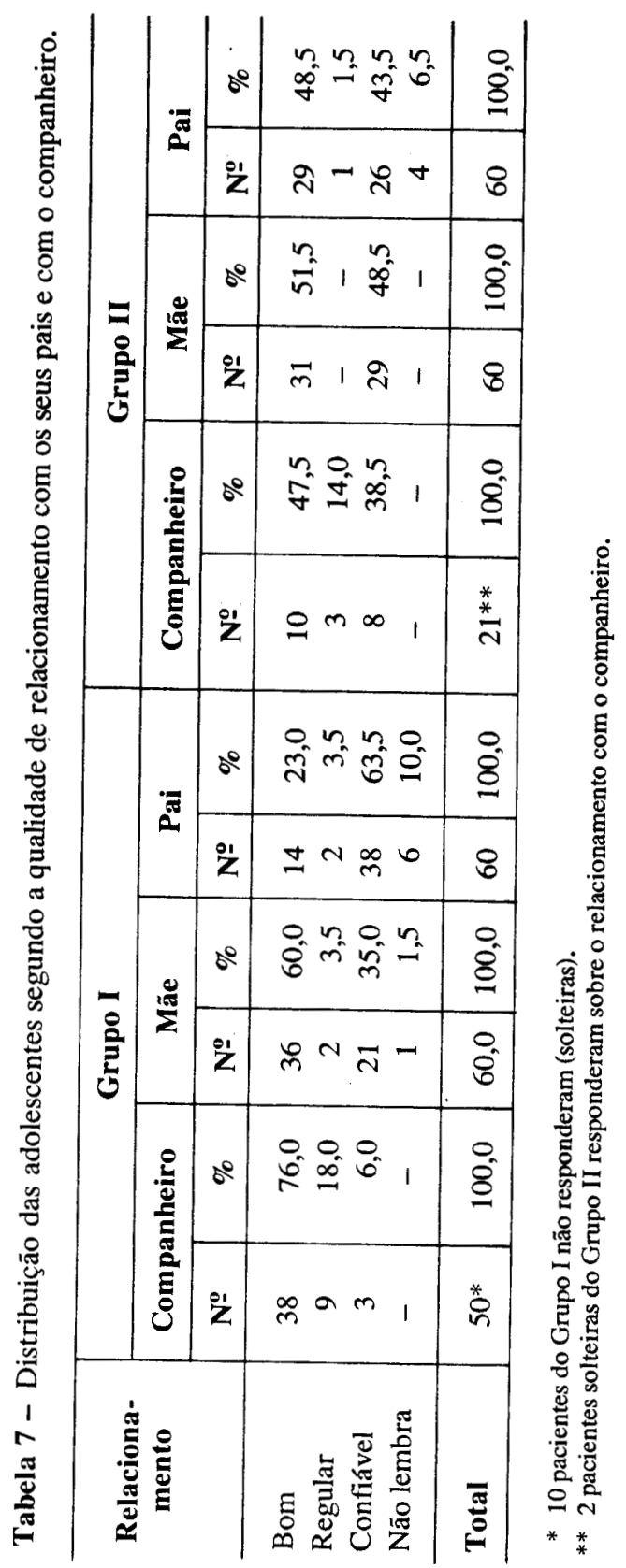




\section{CONCLUSÕES}

Dos dados obtidos na pesquisa, podemos concluir que:

1. Das grávidas, $93,5 \%$ tinha idade entre 15 e 17 anos quando consultaram. E $95 \%$ das grávidas e $82,5 \%$ das não grávidas tinham começado a namorar até, no máximo, a idade de 15 anos. Desses dados, ressalta-se a importância, a nosso ver, de que toda e qualquer orientação que se pretenda junto aos adolescentes deve ser feita antes dos 15 anos de idade. Idealmente, considerando a precocidade sexual da minoria, a orientação deve ser ainda mais precoce, no início da adolescência.

2. Com relação ao número de parceiros, $84 \%$ das grávidas tem ou teve apenas um parceiro, sendo que $80 \%$ delas vivia junto com o companheiro (casadas ou amasiadas). Das não grávidas, $68 \%$ qúé já teve atividade sexual também tem ou teve um único parceiro. Isso faz com que afastemos a idéia, algumas vezes preconizada, da promiscuidade sexual na adolescência pobre, visto que as pacientes que procuram o Hospital das Clínicas são de nível sócio-econômico baixo.

3. É evidente que existe "conhecimento" por parte das adolescentes dos métodos contraceptivos; entretanto, apenas um terço entre as grávidas já os havia utilizado. Porém, a nosso ver, os métodos são pouco conhecidos, pois, do contrário, seriam bem mais usados.

4. Considerando que apenas $47 \%$ das grávidas e $38 \%$ das não grávidas referiram prazer durante o ato sexual, questiona-se a razão da adolescente ter uma vida sexual ativa.

5. É característico, em ambas as populações, uma alta percentagem referir bom relacionamento com os companheiros.

6 . Em relação aos pais, observa-se que existe melhor relacionamento das adolescentes dos dois grupos com a figura materna. $\mathrm{Na}$ população de grávidas, no mesmo nível de proximidade da figura materna está o vínculo conflituoso com a figura paterna.

\section{BIBLIOGRAFIA}

1. ABERASTURY, A. Adolescência. Porto Alegre, Artes Médicas, 1983.

2. ABERASTURY, A. \& KNOBEL, M. Adolescência. Porto Alegre, Artes Médicas, 1981.

3. BARROCO, C. CAMPOS, M. M.; MORAES, M.L.Q.; COSTA, A.O.; BRUSCHINI, C.; PINTO, R. P.; AZEVEDO, C. M.; FERREIRA, N. P.; 
MARQUES, A. C. Gravidez na Adolescência. Brasília, Fundação Carlos Chagas, 1986.

4. BASTOS, A. V. B.; MORRIS, L.; FERNANDES, S. R. P. Saúde a Educação do Jovem - um estudo em Salvador. Salvador, ISP/UFBa, 1989.

5. DEUTSCH, H. Problemas Psicológicos da Adolescência. Rio de Janeiro, Zahar, 1983

6. LANGER, M. Maternidade e Sexo. Porto Alegre, Artes Médicas, 1.981.

7. SOIFER, R. Psicologia da Gravidez, Parto e Puerpério. Porto Alegre, Artes Médicas, 1980.

8. VITIELLO, N. e col. Adolescência Hoje. São Paulo, Roca, 1988. 